\title{
An Intelligent Test Paper Generating Algorithm Based on Maximum Coverage of Knowledge Points
}

\author{
Man Yang \\ Lab of Modern Educational Technology \\ University of Science and Technology of China \\ Hefei, China \\ riemany@mail.ustc.edu.cn
}

\author{
Yinhuang Le, Hui Zhang, Min Wu, Nie Er \\ Lab of Modern Educational Technology \\ University of Science and Technology of China \\ Hefei, China
}

\begin{abstract}
In order to keep up with the requirements of College English Diagnostic Test System developed by our team, after analyzing the pros and cons of current test paper generating algorithms, the MCKP algorithm, which is based on maximum coverage of knowledge points (MCKP), is proposed. In this paper, the knowledge hierarchy of College English Test (CET4) of China is constructed to provide the foundation for MCKP. Then given three main parameters, including the required items number, the mastery rates of knowledge-point and the testing frequencies of knowledge points, the theory and implementation of the MCKP algorithm is elaborated. At last, experiments indicate relatively high success rate and effectiveness of test paper generating process. Therefore, MCKP algorithm can provide an essential improvement in test paper generating process.
\end{abstract}

Keywords- maximum covarage of knowledge points (MCKP); knowledge hierarchy of CET-4;mastery rates ;testing frequencies; success rate;

\section{INTRODUCTION}

With the rapid development of Internet and multimedia technology, more and more English learners in China have tended to use online web-based exercise systems. Generally, these kinds of systems can offer users with amounts of test paper from a pool called item bank. Old exercise systems may probably just pop up a regular test paper. However, equipped with an intelligent test paper generating algorithm, modern systems can generate much more customized test paper that take user individual level into account. To be more specific, a test paper generating algorithm plays as a core part in the exercise system [1]. It can intelligently make the resources into meaningful test paper, which in most cases will focus on the users' drawbacks. Thus, the algorithm will directly affect the learning efficiency for English learners.

Recently, lots of web-based exercise systems have shown their attempts to achieve a new level [2]: supply more convenient, personalized, and high-efficient solution for users, like students, to enhance their study outcomes. As an initial approach to such solution, a web-based learning platform, which pays more attention in interactive and diagnostic performance area, called the College English Diagnostic Exercises System, is developed by our project team, jointed with Anhui USTC iFLYTEK Corporation. In the core part of the system, it is the test paper generating algorithm. Using the concept of adaptive learning, aiming at improving learners' achievements quickly and effectively, combined with the actual needs of learners, an intelligent test paper generating algorithm based on maximum coverage of knowledge points (MCKP) is proposed.

Taking the testing frequencies of knowledge points in the CET-4 examinations of these recent years and the learners' mastery rates of knowledge points into consideration, MCKP algorithm calculates the distribution number of items for every knowledge point and generates papers. In the new papers knowledge points of high mastery rates and low testing frequency are with small proportion and knowledge points of low mastery rates and high testing frequency are with large proportion.

In our strategy, the self-learners only need to set two parameters namely the item number and the threshold of testing frequency of knowledge points, so it simplifies the procedure of selecting questions and improves the success rate. And it can satisfy the different demands of learners with different English levels.

\section{THE CET-4 KNOWLEDGE HIERARCHY}

The knowledge hierarchy of CET-4 is the premise of the exercise system and MCKP algorithm [3]. The hierarchy is shown in Figure 1.

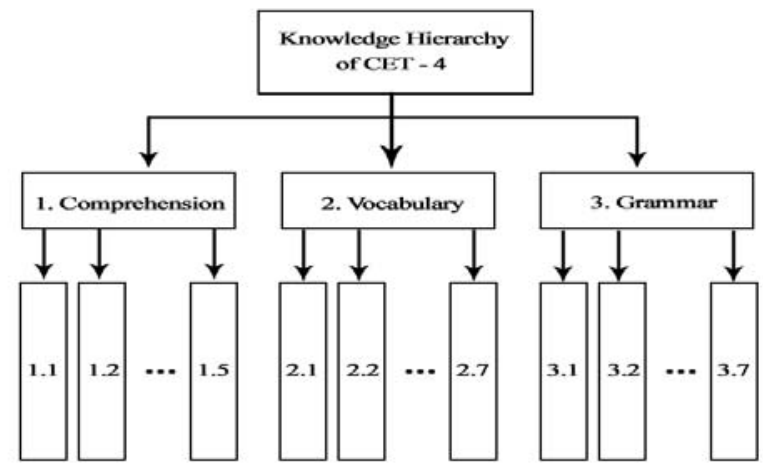

Figure 1. Knowledge hierarchy of CET-4.

After a lot of meticulous research with several English experts, our project team constructs the knowledge hierarchy of CET-4 aiming at carrying on diagnostic evaluation which includes the knowledge points involved with the objective 
questions. The hierarchy is divided into two levels. The first level consists of three knowledge-ability classes which are comprehension, vocabulary and grammar. And every knowledge-ability class includes several knowledge-ability points, or knowledge points for short. According to the knowledge hierarchy, objective items are classified explicitly. The item bank is organized on the basis of the knowledge hierarchy and every item is classified to their knowledge point with the help of several English experts. According to MCKP algorithm, the item number for every knowledge point is calculated, and then corresponding items are selected from the item bank.

\section{DESIGN OF MCKP ALGORITHM}

\section{A. Strategry for Maximum Coverage of Knowledge Points}

MCKP algorithm is a strategy for assigning item to each knowledge point [4] based on the testing frequency of the CET-4 examinations in the recent years and the average mastery rates of knowledge points in the College English Diagnostic Exercise System. The goal of the MCKP algorithm is that knowledge points of high mastery rates and low testing frequency are with small proportion and knowledge points of low mastery rates and high testing frequency are with large proportion. What is more, knowledge points which are rarely involved in the past CET4 exams are not taken into consideration in the first round of distribution before the random correction. When the item number assigned is near to the number set by the user, for the items which cannot be distributed according to the proportional relationship, we use the random function to make adjustments on the distribution of items in every knowledge points.

\section{B. Math Model of Assigning Items in MCKP}

For each knowledge point, when its average testing frequency is larger than the threshold value, items related with it are selected into the new paper in accordance with a certain proportion. The number of items related is concerned with the grasp rate of every knowledge point also. The larger the grasp rate of a certain knowledge point is, the less the items related. Take into consideration these two factors, we proposed a mathematical model for assigning items.

First we use the model to calculate the number needed for each knowledge point [5]. Then we choose items from the item database but this is not in our consideration in this paper. What we do is to calculate the number for every knowledge point. The formula is as follows.

$$
q_{i}=A * \frac{P_{i}}{\sum_{P_{i}>P} P_{i}} *\left(1-x_{i}\right) * N
$$

Parameters explanation:

$P_{i}$ : the average testing frequency of knowledge point $i$;

$P$ : the threshold value set in Step 2;

$N$ : the total item number;

$X_{i}$ : the grasp rate of knowledge point $i$. When $X_{i}$ is very close to 1 , items involved with this knowledge point are not assigned to the new paper; on the contrary when $X_{i}$ is very close to 0 , items involved are assigned a lot.

$A$ : a common value. $A$ is the additional control variable set by our team.

According to the model, during every cycle of assignment the number of items assigned to a certain knowledge point is $q_{i}$.

\section{Process of MCLP Algorithm}

The design steps are described in detail as follows:

- $\quad$ Step 1: Read in the item number set by a learner.

- $\quad$ Step 2: Filter the knowledge points. Some certain knowledge points are rarely appeared in the CET-4 exams. So a threshold can be set to filter the knowledge points. Whether it is too big or too small would affect the item assignment results. First the number of every knowledge point tested during the past years should be added up. And the statistical results are stored in a $1^{*} n$ matrix names $S$. Here $n$ is the number of knowledge points and $a_{i}$ (i is from 1 to $\mathrm{n})$ is the number.

$$
A=\left(\begin{array}{llll}
a_{1} & a_{2} & \cdots & a_{n}
\end{array}\right)
$$

Total number is added up as follows.

$$
T=\sum_{i=1}^{n} a_{i}
$$

The frequency rate of knowledge point $\mathrm{i}$ is

$$
b_{i}=\frac{a_{i}}{T}
$$

Then construct a $1 *$ n matrix to store the frequency rates of every knowledge point.

$$
B=\left(\begin{array}{llll}
b_{1} & b_{2} & \cdots & b_{n}
\end{array}\right)
$$

The threshold would be regularly changed based on the every year's statistical data. According to the testing frequencies of knowledge point during the recent 5 years' CET-4 exams, the frequency of the last third knowledge point is $0.2 \%$, so we set the threshold as $0.2 \%$ that the last three knowledge points is excluded.

- Step 3: Calculate the mastery rate of every knowledge point.

The following formula is applied to calculate the mastery rate of knowledge point $\mathrm{i}$.

$$
M_{i}=\frac{1}{\left(\frac{1-B}{B}\right) \frac{t_{i}}{T_{i}}+1} x_{i}
$$

Where $B$ is a constant between 0 and 1 , according to the sample data testing, set $B$ as $0.9 . M_{i}$ is the grasp rate, $t_{i}$ is the average theoretical time to answer the questions related to knowledge point $i, T_{i}$ is the average practical time to that, $x_{i}$ is the scoring rate. For all the knowledge points, construct a $1^{*}$ n matrix named $M$ to store the mastery rates.

$$
M=\left(\begin{array}{llll}
M_{1} & M_{2} & \cdots & M_{n}
\end{array}\right)
$$

Every learner registered in our system would have his or her matrix. Before the first exercise the value 
of all the elements in the matrix would be zero and with the increase of exercise, the values would be updated.

- $\quad$ Step 4: Assign items to every knowledge point on the basis of the following formula.

In the first cycle, the item number assigned to knowledge point $i(i$ is between 1 and 19) is:

$$
q_{i}=A * \frac{P_{i}}{\sum_{P_{i}>P} P_{i}} *\left(1-x_{i}\right) * N
$$

After one cycle, the total number of items is Q.

$$
Q=\sum_{i=1}^{n} q_{i}
$$

If $Q$ is not equal to $N$, then carry on the next distribution in accordance with the following method.

If $Q$ is smaller than $N$, then the next cycle of distribution continues on. Now the total number to be assigned is $N-Q$. In the current cycle of distribution, the item number assigned to every knowledge point $i$ is

$$
q_{i}=A^{*} \frac{P_{i}}{\sum_{P_{i}>P} P_{i}} *\left(1-X_{i}\right) *(N-Q)
$$

If $\mathrm{Q}$ is larger than $\mathrm{n}$, then it is the time to delete items. The items needed to be deleted are $\mathrm{Q}-\mathrm{N}$. The number for every knowledge point is:

$$
q_{i}=A^{*} \frac{P_{i}}{\sum_{P_{i}>P} P_{i}} *\left(1-x_{i}\right) *(Q-N)
$$

Alternately executing the above two processes, the cycle of assigning and deleting items would not be stopped until the $Q$ equals $N$ or the items could not be selected. "The items could be selected" means that the number of cycles is equal to $N$ which is the total number of item to be selected.

- $\quad$ Step 5: Random correction. After Step 4, if $Q$ is still not equal to $N$, then use the method of random correction to add or delete items for a random chosen knowledge point until $Q$ is equal to $N$. The process for the method consists of $N$ - $Q$ cycles, or $Q$ $-N$ cycles if $Q$ is larger than $N$. In one cycle, use the random function to choose a knowledge point randomly. Then add or delete(if $Q$ is larger than $N$ ) one item for the selected knowledge point. After that enter into the next cycle. Stop while $Q$ is equal to $N$.

\section{Implementation of MCLP Algorithm}

In the previous section, the whole process has been amply elaborated. Where there is an idea, there is a model. Hence, in this section the implementation model is proposed to achieve the algorithm of maximum coverage of knowledge points.

The implementation consists of three parts. Most of the pseudo codes followed are discussed a lot in the process design. The first is to calculate the testing frequency of every knowledge point by calling the function frequency(). The second is to calculate the grasp rate of every knowledge point by calling the function kpmastery(). As the details of the calculation is elaborated in Step 2 and Step 3 of Part III and they are not the key of MCKP algorithm, so we don't provide the pseudo codes. The last but the most important step is to calculate the assigned number for every knowledge point. Pseudo codes of the third part are as follows.

selectItem(itemNum, freq $[\mathrm{m}]$, mastery $[\mathrm{m}]$, threshold)

Input:

Prompt the user for the number of items: $\mathrm{N}$

\section{Initialization:}

Set freq $[\mathrm{m}] \leftarrow$ frequency () ; mastery $[\mathrm{m}] \leftarrow$ kpmastery () ;

Set itemNum $\leftarrow \mathrm{n}$; itemSum $=0$; itemArray $[\mathrm{m}]=0$;

Set tempSum $=0$; flag $=0$; cycleCnt $=0$;

\section{Iteration:}

1: While flag $==0$ AND cycleCnt $<=$ itemNum do

2: If itemSum $<=$ itemNum Then

3: $\quad$ For i from 1 to length of freq[m] step 1 do

4: $\quad$ If mastery[i] $>$ threshold.

5: Then increase itemArray[i] and tempSum in the

6: current assigned number of itemNum -itemSum

7: $\quad$ End if

8: $\quad$ End for

9: Else

10: For $i$ from 1 to length of freq[m] step 1 do

11: If mastery[i] $>$ threshold

12: Then decrease itemArray[i] and tempSum in the

13: $\quad$ current deleted number of itemSum -itemNum

14: $\quad$ End if

15: End for

16: End if

17: If itemSum $==$ itemNum

18: $\quad$ Then flag $\leftarrow 1$

19: End if

20: $\quad$ itemSum $\leftarrow$ tempSum

21: $\quad$ cycleCnt $\leftarrow$ cycleCnt +1

22: End while

23: While itemSum !=itemNum do

24: Choose a knowledge point i randomly

25: If itemSum > itemNum Then

26: $\quad$ itemArray $[\mathrm{i}] \leftarrow$ itemArray $[\mathrm{i}]-1$

27: $\quad$ itemSum $\leftarrow$ itemSum -1

28: Else

29: $\quad$ itemArray $[\mathrm{i}] \leftarrow$ itemArray $[\mathrm{i}]+1$

30: $\quad$ itemSum $\leftarrow$ itemSum +1

31: End if

32: End while

33: Output matrix itemArray

Figure 2. The pseudo codes of selectItem().

\section{EXPERIMENT AND TEST}

Having gone through MCKP design and implementation model, it is high time to bring the model into reality.

In order to confirm the superiority and validity, the MCKP algorithm is run in MATLAB 7.8. The platform is a 
2.7GHz Intel Dual-Core machine running Windows 7 premium with 3GB RAM. The item number is set as 100 . The number for new test papers is set as 282. The threshold value for frequency of knowledge point is set as 0.002 . At last, a result of 279 new papers is achieved, which suggests that the success rate is $98.9 \%$. And the average running time of calculating the number for all the knowledge points is less than 5 seconds. The table is as follows.

TABLE I. PERFORMANCE OF MCKP ALGORITHM

\begin{tabular}{|l|c|c|}
\hline \multirow{2}{*}{} & \multicolumn{2}{|c|}{ Measurements } \\
\cline { 2 - 3 } MCKP & Success rate & Running time \\
\hline & $98.9 \%$ & 5senonds \\
\hline
\end{tabular}

As it shows in the table, it is proved that the MCKP can improve not only the success rate, but also the efficiency of generating papers.

\section{CONCLUSIONS}

To satisfy the individual requirements of different learners and diagnose the learning ability, this paper proposes a test paper generating algorithm which takes both the mastery rate of knowledge point and the testing frequency of knowledge point into consideration. It is particularly useful for generating test papers online for webbased exercise system. First the knowledge hierarchy of CET-4 is introduced [7]. And then in the premise of this, we discuss in detail the design and implementation of the algorithm while we test the mastery rate of knowledge points. At last we evaluated the performance of the proposed approach. The performance results have shown that the MCKP not only achieved good success rate, but also a fast runtime. Thus the proposed algorithm is effective for online web-based individual test paper generation in terms of runtime efficiency and success rate.

\section{ACKNOWLEDGMENT}

The research is supported by the Project of College Teaching and Research at the provincial level of Anhui Province, 2010.

\section{REFERENCES}

[1] Weijun Liu, Tian-Qi Yuan, Jiang-Wen Xiao and Zhiwei Wang, "Design and Implementation of Test Paper Auto-Generation Strategy for Electric Energy Measurement,” Proc. IEEE Symp. Interational Conference on Intelligent Control and Information Processing(ICICIP 11), IEEE Press, Nov. 2011, pp. 1013-1017,doi: 10.1109/ ICICIP.2011.6008404.

[2] Zhen-hua Jia, Chun-e Zhang and Hao-shuai Fang, "The Research and Application of General Item Bank Automatic Test Paper Genaration Based on Improved Genetic Algorithm," Proc. IEEE Symp. Internatinal Conference on Computing, Control and Industial Engineering(CCIENG 11), IEEE Press, Jan. 2011, pp. 14-18, doi: 10.1109/CCIENG.2011.6007945.

[3] Luo jia, Zhang Renjin and Jin yi, "Implementation Strategy of Several Key Issues in Intelligent Testing Paper Generation System,” Proc. IEEE Symp. Interational Conference on Computatinal Intelligence and Software Engineering(CISE 09), IEEE Press, Jan. 2009, pp. doi: 10.1109/CISE.2009.5363420.

[4] Yongjun Wang and Yuyu Huang, "Intelligent Arithmetic of Test Paper Forming Based on Question Style and Grade of Knowledge,” Computer Applications and Software, vol. 21, Aug. 2004, pp. 111113.

[5] Lin, B.M.T., Gwo-Jen Hwang and Tsung-Liang Lin, "An effective approach for test-sheet composition with large scale item banks,” Computer \& Education, vol.46, Feb. 2006, pp. 122-139, doi: 10.1016/j.compedu.2003.11.004.

[6] Gwo-Jen Hwang, Hui-Chun Chu, Peng-Yeng Yin and Ji-Yu Lin, "An innovative parallel test sheet composition approach to meet multi pleassessment criteria for national tests," Computer \& Education, vol.51, Nov. 2008, pp.1058-1072, doi: 10.1016/j.compedu.2007. 10.006 .

[7] Minh Luan Nguyen, Siu Cheung Hui and ALvis C.M. Fong, "An Efficient Multi-Objective Optimization Approach for Online Test Paper Generation,” Proc. IEEE Symp. Symposium on COmputatinal Intelligence in Multicriteria Decision-Making(MDCM 11), IEEE Press, Jan. 2011, pp. 182-189, doi: 10.1109/SMDCM.2011.5949277. 